\title{
Avaliação da sensibilidade dolorosa após tratamento endodôntico com limas WaveOne gold e ProGlider, com e sem preparo prévio dos terços cervical e médio
}

\section{antes da patência foraminal}

\author{
Assessment of painful sensitivity after endodontic treatment with WaveOne gold and ProGlider \\ files, with and without prior preparation of the cervical and middle thirds before foraminal patency \\ Evaluación de la sensibilidad dolorosa después del tratamiento endodóntico con limas WaveOne
}

Gold y Proglider, con y sin preparación previa de los tercios cervical y medio antes de la permeabilidad foraminal

Recebido: 31/05/2021 | Revisado: 10/06/2021 | Aceito: 13/06/2021 | Publicado: 26/06/2021

Fabrício da Silva Santos

ORCID: https://orcid.org/0000-0003-2806-6207 Faculdade São Leopoldo Mandic, Brasil

E-mail: drfabricio.83@gmail.com

Ana Grasiela da Silva Limoeiro

ORCID: https://orcid.org/0000-0003-4633-720X Faculdade de Ilhéus, Brasil

E-mail: grasielaimoeiro@gmail.com

Alexandre Sigrist De Martin

ORCID: https://orcid.org/0000-0002-3320-9172

Faculdade São Leopoldo Mandic, Brasil

Email: a-sigrist@uol.com.br

Carlos Eduardo Fontana

ORCID: https://orcid.org/0000-0002-2868-6839

Faculdade PUC Campinas, Brasil

E-mail: ceffontana@hotmail.com

Sérgio Luiz Pinheiro

ORCID: https://orcid.org/0000-0001-7157-4923

Faculdade PUC Campinas, Brasil

E- mail: slpinho@hotmail.com

Rina Andrea Pelegrine

ORCID: https://orcid.org/0000-0003-4175-2121

Faculdade São Leopoldo Mandic, Brasil

E-mail: rinapelegrine@terra.com.br

Daniel Guimarães Pedro Rocha

ORCID: https://orcid.org/0000-0001-9792-2260

Faculdade São Leopoldo Mandic, Brasil

E-mail: dnlrocha@uol.com.br

Wayne Martins Nascimento

ORCID: https://orcid.org/0000-0003-4201-4710

Faculdade São Leopoldo Mandic, Brasil

E-mail: waynemartinsn@ hotmail.com

Carlos Eduardo da Silveira Bueno

ORCID: https://orcid.org/0000-0002-2675-0884

Faculdade São Leopoldo Mandic, Brasil

E-mail: carlosesbueno@terra.com.br

\begin{abstract}
Resumo
Este trabalho teve como objetivo avaliar a sintomatologia dolorosa pós-tratamento endodôntico, em sessão única, em dentes unirradiculares com necrose pulpar, variando duas técnicas de patência do forame: com e sem preparo prévio dos terços cervical e médio. Os pacientes foram divididos de forma aleatória em dois grupos ( $\mathrm{n}=23$ ). Grupo SSP (Sem Preparo Prévio): o tratamento endodôntico foi realizado com limas Wave One Gold (Dentsply Sirona, Ballaigues Suíça) associado à patência com ProGlider (Dentsply Sirona, Ballaigues - Suíça) previamente ao preparo dos terços cervical e médio. Grupo CPP (Com Preparo Prévio): tratamento endodôntico foi realizado com limas Wave One Gold associada à patência com ProGlider após o preparo dos terços cervical e médio. A irrigação foi feita com $\mathrm{NaOCl} 2,5 \%$ e os canais foram obturados pela técnica do cone único com cimento AH Plus, utilizando o compactador de
\end{abstract}


McSpadden. Após o tratamento os pacientes responderam a um questionário com a escala de dor EVA, e classificando de 0 a 10, nos períodos de 24 horas, 72 horas, e 1 semana pós-tratamento. Os dados foram analisados através dos testes de Mann-Whitney, Kruskal-Wallis (Student-Newman-Keuls) teste de Dunn e teste Qui-quadrado. Os resultados mostraram que nos pacientes em que foi realizado previamente o preparo dos terços cervical e médio, não houve diferenças estatísticas entre os escores de dor nos diferentes tempos experimentais $(\mathrm{p}=0.328)$. A comparação de dor após 24 horas e 72 horas apresentou valores estatisticamente semelhantes $(\mathrm{p}=0.384)$, o mesmo acontecendo com a comparação de 72 horas com uma semana $(\mathrm{p}=1.00)$. O grupo sem preparo prévio revelou diferenças significantes entre os resultados $(\mathrm{p}=0.046)$. O teste complementar de Dunn mostrou que as diferenças se limitavam aos escores de dor após 24 horas e os resultados após uma semana ( $\mathrm{p}=0.042)$. O teste de Mann-Whitney, aplicado aos dados obtidos em ambos os grupos experimentais, revelou que nos tempos de 24 horas $(p=0.562), 72$ horas $(p=1.00)$ e 1 semana $(\mathrm{p}=1.00)$, não houve diferenças entre os tipos de preparo realizados. Concluiu-se que os níveis de dor pós-tratamento endodôntico em dentes unirradiculares com necrose pulpar com ou sem preparo prévio do terço cervical e médio do canal apresentou comportamento equivalente.

Palavras-chave: Dor pós-endodôntica; Dor pós-operatória; Patência apical.

\begin{abstract}
This study aimed to evaluate painful symptoms after endodontic treatment, in a single session, on uniradicular teeth with pulp necrosis, varying two techniques of foramen patency: with and without previous preparation of the cervical and middle thirds. Patients were randomly divided into two groups $(n=23)$. SSP Group (Without Previous Preparation): the endodontic treatment was performed with Wave One Gold files (Dentsply Sirona, Ballaigues Switzerland) associated with the patency with ProGlider (Dentsply Sirona, Ballaigues - Switzerland) prior to the preparation of the cervical and middle thirds. CPP Group (with Previous Preparation): endodontic treatment was performed with Wave One Gold files associated with patency with ProGlider after the preparation of the cervical and middle thirds. Irrigation was carried out with $2.5 \% \mathrm{NaOCl}$ and the channels were filled using the single cone technique with AH Plus cement, using the McSpadden compactor. After treatment, the patients answered a questionnaire with the VAS pain scale, and rated it from 0 to 10, in the periods of 24 hours, 72 hours, and 1 week after treatment. The data were analyzed using the Mann-Whitney, Kruskal-Wallis (Student-Newman-Keuls) Dunn test and Chi-square test. The results showed that in patients who had previously prepared the cervical and middle thirds, there were no statistical differences between pain scores at different experimental times $(\mathrm{p}=0.328)$. The comparison of pain after 24 hours and 72 hours showed statistically similar values $(\mathrm{p}=0.384)$, as did the comparison of 72 hours with one week $(\mathrm{p}=1.00)$. The group without prior preparation revealed significant differences between the results ( $\mathrm{p}$ $=0.046)$. Dunn's complementary test showed that the differences were limited to pain scores after 24 hours and the results after one week $(\mathrm{p}=0.042)$. The Mann-Whitney test, applied to data obtained in both experimental groups, revealed that at 24 hours $(p=0.562), 72$ hours $(p=1.00)$ and 1 week $(p=1.00)$, there were no differences between the types of preparation performed. It was concluded that the levels of pain after endodontic treatment in uniradicular teeth with pulp necrosis with or without previous preparation of the cervical and middle third of the canal presented an equivalent behavior.
\end{abstract}

Keywords: Apical patency; Postendodontic pain; Postoperative pain.

\title{
Resumen
}

Este estudio tuvo como objetivo evaluar los síntomas dolorosos tras el tratamiento endodóntico, en una sola sesión, en dientes unirradiculares con necrosis pulpar, variando das técnicas de permeabilidad del foramen: con y sin preparación previa de los tercios cervical y medio. Los pacientes se dividieron aleatoriamente en dos grupos $(n=23)$. Grupo SSP (sin preparación previa): el tratamiento de endodoncia se realizó con limas Wave One Gold (Dentsply Sirona, Ballaigues - Suiza) asociadas a la permeabilidad con ProGlider (Dentsply Sirona, Ballaigues - Suiza) antes de la preparación de los tercios cervical y medio. Grupo CPP (con preparación previa): el tratamiento de endodoncia se realizó con limas Wave One Gold asociadas a la permeabilidad con ProGlider después de la preparación de los tercios cervical y medio. El riego se realizó con $\mathrm{NaOCl}$ al $2.5 \%$ y los canales se rellenaron mediante la técnica de cono único con cemento AH Plus, utilizando el compactador McSpadden. Después del tratamiento, los pacientes respondieron un cuestionario con la escala de dolor EVA, y lo calificaron de 0 a 10, en los períodos de 24 horas, 72 horas y 1 semana después del tratamiento. Los datos se analizaron mediante la prueba de Dunn de Mann-Whitney, Kruskal-Wallis (Student-Newman-Keuls) y la prueba de Chi-cuadrado. Los resultados mostraron que en pacientes que previamente habían preparado los tercios cervical y medio, no hubo diferencias estadísticas entre las puntuaciones de dolor en diferentes momentos experimentales $(\mathrm{p}=0.328)$. La comparación de dolor a las 24 horas y 72 horas mostró valores estadísticamente similares $(\mathrm{p}=0.384)$, al igual que la comparación de 72 horas con una semana $(\mathrm{p}=1.00)$. El grupo sin preparación previa reveló diferencias significativas entre los resultados $(\mathrm{p}=0.046)$. La prueba complementaria de Dunn mostró que las diferencias se limitaron a las puntuaciones de dolor después de 24 horas y los resultados después de una semana $(\mathrm{p}=0.042)$. La prueba de Mann-Whitney, aplicada a los datos obtenidos en ambos grupos experimentales, reveló que a las 24 horas $(\mathrm{p}=0.562), 72$ horas $(\mathrm{p}=1.00)$ y 1 semana $(\mathrm{p}=1.00)$, no hubo diferencias entre los tipos. de preparación realizada. Se concluyó que los niveles de dolor tras el tratamiento endodóntico en 
dientes unirradiculares con necrosis pulpar con o sin preparación previa del tercio cervical y medio del canal presentaron un comportamiento equivalente.

Palabras clave: Permeabilidad apical; Dolor postendodóntica; Dolor posoperatoria.

\section{Introdução}

A terapia endodôntica é o procedimento que envolve o tratamento da polpa dental com objetivo de manter o dente natural (Junior et al., 2016). O preparo químico-mecânico tem o objetivo de eliminar microrganismos, limpando e modelando o sistema de canais radiculares, removendo material orgânico e inorgânico (Shilder, 1967). Até a década de 1980, era preconizado a técnica de preparo ápice/coroa, responsável pela alta incidência da dor pós-operatória como as periodontites apicais agudas. A partir de 1980, Marshal e Pappin (1980) propuseram um novo conceito de preparo denominado coroa-ápice, com a utilização de instrumentos endodônticos mais calibrosos nos terços cervical e médio, com avanço gradativo em direção apical diminuindo-se o diâmetro das limas (Técnica de OREGON). Dessa maneira, minimizou-se a extrusão de debris, reduzindo de forma eficaz os riscos de dor pós-operatória.

Atualmente, instrumentos mecanizados como o sistema WaveOne Gold (Dentsply Maillefer, Ballaigues, Suiça), utilizam uma liga de NiTi resistente e flexível, devido ao seu tratamento térmico gold. Esse sistema permite que se realize todo o tratamento endodôntico com apenas uma lima (Larsen et al., 2009).

A desinfecção dos canais radiculares pode ser potencializada pela manutenção da patência apical, definida como o procedimento no qual a porção apical do canal radicular é mantida livre de detritos pela recapitulação com uma lima, levada até a constrição apical, sem, no entanto, ampliá-la (Buchanan, 1989). A limpeza do forame apical cria melhores condições biológicas para que ocorra o processo de reparo. Em casos de necrose pulpar, recomenda-se a passagem de um instrumento além do forame, para que o canal seja completamente limpo (Souza et al., 2000).

ProGlider (Dentsply Maillefer, Ballaigues, Suíça) é uma lima de NiTi M-Wire, utilizada na exploração e préalargamento de canais com eficiência e segurança, devido sua secção quadrada, com uma ponta semiativa e taper progressivos de $2 \%$ a $8 \%$ ao longo do seu comprimento, o que garante a flexibilidade e resistência a fadiga cíclica, reduzindo o risco de fratura do instrumento.

A dor pós-operatória é uma das intercorrências do tratamento endodôntico e pode estar associada as injúrias mecânicas, químicas e/ou microbiológicas aos tecidos periapicais durante o preparo químico-mecânico (Sathorn et al., 2008; Arias et al., 2009). Durante a instrumentação dos canais radiculares, todas as técnicas podem causar extrusão de debris, mesmo quando se usa o movimento reciprocante e a instrumentação coroa-ápice (Arias et al., 2009; Arias et al., 2013). O objetivo desse estudo clínico prospectivo foi avaliar in vivo a dor pós-endodôntica, utilizando o sistema WaveOne Gold associado a lima ProGlider antes ou após o preparo prévio dos terços cervical e médio, nos períodos de 24 horas, 72 horas e uma semana após os tratamentos endodônticos. A hipótese nula é que não haveria diferença entre as duas técnicas aplicadas (patência com preparo prévio e sem preparo prévio dos terços cervical e médio) quanto a sintomatologia dolorosa.

\section{Metodologia}

Após aprovação do Comitê de Ética local (1.829.704), todos os voluntários convidados a participar deste estudo clínico foram esclarecidos dos procedimentos, bem como riscos e benefícios, e seu direito a sua livre escolha em relação à participação. Um consentimento por escrito foi assinado e uma cópia foi entregue a todos os voluntários. 


\section{Cálculo Amostral}

Para se obter o número de amostras necessárias para comparar a dor pós-operatória, foi realizado o cálculo amostral, no qual adotou-se um erro tipo I ( $\alpha$ ) de $5 \%$, um erro tipo II ( $\beta$ ) de $20 \%$, um poder de $80 \%$ e os efeitos (tamanhos de efeito $=\mathrm{d}$ de Cohen) verificados para uma amostra aleatória de 10 pacientes por grupo experimental. A partir de dados obtidos, procedeuse o teste amostral para amostras pareadas e não pareadas e encontrou-se um dos grupos experimentais. $\mathrm{O}$ tamanho amostral final foi, portanto, de 46 pacientes selecionados dentro de um universo de 120 pacientes atendidos durante um período de um ano pelo pesquisador. O número amostral necessário de 21 pacientes para amostras pareadas, e de 23 para amostras não pareadas para cada.

\section{Seleção dos pacientes}

Quarenta e seis pacientes voluntários de ambos os gêneros, com faixa etária entre 18 a 60 anos, foram incluídos neste estudo, de acordo com os critérios definidos. Todos os tratamentos foram realizados em um consultório particular, em sessão única, por um único operador especialista em endodontia. Os pacientes receberam um Termo de Consentimento Livre e Esclarecido em duas vias (uma do paciente e outro do pesquisador).

Critérios de Inclusão: Pacientes com necessidade de tratamento endodôntico com necrose pulpar, presença ou ausência de imagem compatível com lesão periapical, sem sintomatologia dolorosa e que não estivesse fazendo uso de nenhuma medicação há pelo menos 10 dias (analgésicos, anti-inflamatório, antibiótico); sem tratamento endodôntico prévio; canais não calcificados e com forame formado e diâmetro apical entre \#10 e \#20. Canais com curvatura máxima de 30 graus pelo método de Pruett (1997) considerando ângulo e raio de curvatura. Foi realizada anamnese, exame clínico e radiográfico, realizando teste de sensibilidade com gás refrigerado (Endo-frost: Coltene Whale-dent, Langenau - Germany) através do uso de uma bolinha de algodão colocada no terço médio da superfície vestibular do elemento dental a ser tratado. Após 10s se não houvesse resposta, o teste seria considerado negativo.

Critérios de Exclusão: Canais com diâmetro menor que \#10 ou maior que \#20, uso de medicamentos recentemente, presença de retratamentos endodônticos, edema, canais calcificados, raízes com curvatura acentuada superior a $30^{\circ}$ e que responderam positivamente ao teste de sensibilidade pulpar com spray refrigerante foram excluídos.

Os pacientes foram divididos de forma aleatória (https://www.random.org) em dois grupos ( $n=23)$ : Grupo SPP tratamento endodôntico foi realizado com limas do sistema WaveOne Gold (Dentsply Maillefer, Ballaigues - Suíça) associadas a ProGlider (Dentsply Maillefer, Ballaigues - Suíça) previamente ao preparo dos terços cervical e médio; Grupo CPP em que o tratamento endodôntico foi realizado com limas do sistema WaveOne Gold associadas a ProGlider após o preparo dos terços cervical e médio.

\section{Protocolo de Tratamento}

Após o planejamento para realização dos tratamentos, foi realizada raspagem supragengival e profilaxia do elemento dental a ser tratado. Posteriormente, anestesia infiltrativa com 3,6 mL de Lidocaína com 1:100.000 de epinefrina (DFL, Rio de Janeiro, RJ - Brasil), o acesso cirúrgico foi realizado com broca diamantada 1014 (KG Sorensen - São Paulo - Brasil) e remoção do teto com broca de ponta inativa 3082 (KG Sorensen - São Paulo - Brasil), seguindo a forma de contorno e conveniência da cavidade. A irrigação foi realizada com Hipoclorito de sódio 2,5\% e exploração do canal radicular em 2/3 do seu comprimento com limas Kerr \#10 ou Kerr \#15 (Dentsply Maillefer, Ballaigues - Suíça).

Após a exploração inicial do canal, o preparo biomecânico foi realizado com as limas WaveOne Gold selecionada de acordo com a amplitude do canal radicular. A instrumentação foi realizada com o motor X-Smart Plus (Dentsply Maillefer, Ballaigues - Suíça) no modo Wave One Gold, de acordo com a técnica recomendada pelo fabricante (movimentos curtos de 
entrada e saída com pincelamento em direção apical).

\section{Grupo CPP}

Inicialmente realizou-se uma irrigação com $5 \mathrm{~mL}$ de hipoclorito de sódio 2,5\% (Asfer Indústria Química Ltda, São Caetano do Sul - SP - Brasil) na embocadura do canal, em seguida, utilizando a lima Wave One Gold (Primary 25.07 ou Medium 35.06 dependendo da amplitude do canal) instrumentou-se a região do terço coronário. Uma nova irrigação com $5 \mathrm{~mL}$ de NaOCL 2,5\% foram realizadas até o comprimento instrumentado com agulhas NaviTip 31G (Ultradent do Brasil, Indaiatuba - SP). Preparo do terço médio com WaveOne Gold e novamente irrigação com $5 \mathrm{~mL} \mathrm{NaOCl} \mathrm{2,5 \% .} \mathrm{Odontometria}$ eletrônica com auxílio do localizador foraminal Bingo (Forum Engineering Technologies, Rishon Le-Zion, Israel) e lima kerr \#15. Nova irrigação com $5 \mathrm{~mL}$ NaOCL 2,5\% e patência do forame com lima ProGlider (Dentsply Maillefer, Ballaigues Suíça) $1 \mathrm{~mm}$ além do comprimento real do dente, ou seja, $1 \mathrm{~mm}$ além do forame apical. Nova irrigação com $5 \mathrm{~mL}$ NaOCL 2,5\% e preparo do terço apical com Wave One Gold selecionada de acordo com o conduto (25.07 ou 35.06).

\section{Grupo SPP}

A Glide Path foi realizada com a lima ProGlider diretamente após acesso cirúrgico, irrigação com $5 \mathrm{~mL}$ de $\mathrm{NaOCl}$ 2,5\% e a odontometria eletronica (Forum Engineering Technologies, Rishon Le-Zion, Israel), sem nenhum tipo de preparo prévio nos terços cervical e médio, da seguinte maneira: Irrigação com $5 \mathrm{~mL}$ de $\mathrm{NaOCl}$ 2,5\% com agulha NaviTip 30G e exploração do canal com limas Kerr \#10. A irrigação foi realizada com $5 \mathrm{~mL} \mathrm{NaOCl}$ 2,5\% e odontometria eletrônica. Realizado a Glide path com a lima ProGlider $1 \mathrm{~mm}$ além do forame apical, irrigação com hipoclorito de sódio 2,5\%, introdução da lima WaveOne Gold (25.07 ou 35.06 de acordo com a amplitude do canal) com 3 movimentos em pequena amplitude de entrada e saída com pincelamento (de acordo com o fabricante). Nova irrigação com $5 \mathrm{~mL} \mathrm{NaOCl}$ 2,5\% até o terço instrumentado. Preparo do terço médio com WaveOne Gold. Irrigação com $5 \mathrm{~mL} \mathrm{NaOCl}$ 2,5\% até o terço médio. Terceira penetração da lima WaveOne Gold até o comprimento de trabalho.

\section{Irrigação Final e Secagem}

$\mathrm{Na}$ irrigação final foram utilizados $5 \mathrm{~mL} \mathrm{NaOCl} 2,5 \%$ ativando a solução em 3 ciclos de 20s com ponta plástica Easy Clean no modo recíproco 2mm aquém do comprimento de trabalho. Em seguida, 3 ciclos com EDTA 17\% (Fórmula \& Ação, São Paulo - SP, Brasil) com ativação com pontas plástica Easy Clean para remoção da smear-layer. O protocolo com hipoclorito de sódio 2,5\% foi repetido e uma irrigação final com $5 \mathrm{~mL}$ de soro fisiológico 0,9\% (Eurofarma do Brasil, Ribeirão Preto-SP) com agulha de $30 \mathrm{G}, 3 \mathrm{~mm}$ aquém do comprimento de trabalho. A secagem dos canais foi feita com ponta de papel absorvente de acordo com o calibre da lima final (25.07 ou 35.06) para posteriormente iniciar o processo de obturação.

\section{Obturação}

Após o preparo químico-mecânico e a secagem dos canais, os dentes dos dois grupos foram obturados com cone de guta percha do sistema correspondente a lima final utilizada com termoplastificação com compactador MacSppaden (Dentsply Maillefer, Ballaigues - Suíça) tamanho 50 a $5 \mathrm{~mm}$ aquém do comprimento de trabalho. O cimento utilizado foi o AH Plus (Dentsply Maillefer, Ballaigues - Suíça). O excesso de guta percha foi removido com auxílio do Condensador de Paiva $\mathrm{n}^{\circ} 3$ (Golgran Indústria Comércio de Instr Odontológicos, São Caetano do Sul, SP - Brasil) aquecido. Radiografia periapical foi realizada para avaliar a qualidade da obturação. Concluída a obturação do canal, a limpeza final (remoção de cimento das paredes dentinárias), a proteção com coltosol da entrada do canal radicular, restauração provisória com resina composta Liss (FGM Produtos Odontológicos, Joinville, SC - Brasil) e ajuste oclusal foram realizados. 


\section{Controle pós-tratamento}

Após o tratamento, todos os participantes receberam um questionário baseado em uma escala visual analógica (EVA) para registrar sua avaliação da dor após 24 horas, 48 horas, 72 horas e 1 semana. A EVA, validada em artigos anteriores (Myles et al., 1999), consiste em uma linha horizontal medindo $100 \mathrm{~mm}$ de comprimento ao longo da qual os valores numéricos foram agrupados em categorias visuais. Os pacientes foram instruídos a atribuir um valor à sua dor percebida com base na EVA.

O controle foi realizado por um único profissional que entrou em contato com os pacientes através de ligações telefônicas, nos intervalos de 24, 72 horas e uma semana após obturação dos canais. Primeiro foi questionado aos participantes da pesquisa sobre a presença ou ausência de dor pós-operatória, a intensidade da dor e a necessidade do uso de medicações analgésicas ou anti-inflamatórias. Caso necessário, os pacientes foram orientados para o uso de Ibuprofeno 600mg no intervalo de 8 em 8 horas. Foi também pedido aos pacientes que marcassem um ' $X$ " na Escala Visual Analógica (EVA) de acordo com sua percepção de dor, nos mesmos intervalos de tempo. Para isso o paciente levou 3 folhas contendo a EVA, fazendo as marcações nos períodos de 24 horas, 72 horas e uma semana após a realização do tratamento. A EVA é uma escala simples em que existe um ponto inicial que representa ausência de dor e um ponto final representando dor extrema. Os resultados obtidos a partir do registro dos escores atribuídos pelos pacientes à escala visual analógica foram submetidos ao teste de normalidade de Shapiro-Wilk, revelando uma distribuição não-paramétrica dos dados.

O teste de Kruskal-Wallis (Student-Newman-Keuls) complementado pelo teste de Dunn foi utilizado para detectar diferenças significantes intragrupos, em nível de significância de 5\%. O teste de Mann-Whitney foi utilizado com o mesmo nível de significância na deteç̧ão de diferenças intergrupos. O teste Qui-quadrado (com correção de continuidade de Yates) foi utilizado nas comparações intragrupo, enquanto o teste Qui-quadrado (exato de Fisher) foi utilizado entre grupos, observandose os mesmos tempos experimentais.

\section{Resultados}

No grupo onde foi realizado o preparo cervical prévio, 4 dos 23 pacientes $(17,4 \%)$ relataram algum tipo de dor ou desconforto nas primeiras 24 horas, sendo o menor escore igual a 1 e o maior igual a 2. Dentre esses 4 pacientes, um utilizou o ibuprofeno (4,3\% do total), sendo seu uso descontinuado após este período. Após 72 horas do procedimento, dois pacientes relataram dor ou desconforto ( $8,7 \%$ do total), classificando como escores 1 e 3. Destes, apenas um utilizou o analgésico (4,3\% do total), continuando seu uso após uma semana do procedimento, relatando remissão da sintomatologia.

No grupo onde não foi feito o preparo cervical prévio, 5 dos 23 pacientes (21,7\%) relataram algum tipo de dor ou desconforto nas primeiras 24 horas, sendo o menor escore igual a 1 e o maior igual a 7. Dentre esses 5 pacientes, o paciente que relatou o maior escore de dor utilizou o ibuprofeno (4,3\% do total), sendo seu uso descontinuado nos períodos de avaliação subsequentes. Após 72 horas do procedimento, dois pacientes relataram dor ou desconforto (8,7\% do total), classificando como escores 1 e 2, sendo que nenhum precisou fazer uso de analgésico. Após uma semana do procedimento, nenhum paciente relatou dor ou fez uso de analgésico (Gráfico 1). 
Gráfico 1. O gráfico 1 mostram as porcentagens de pacientes que reportaram escores de dor pós-operatória moderada (4 a 7), leve (1 a 3) ou ausência de dor (0) nos tempos experimentais estudados.

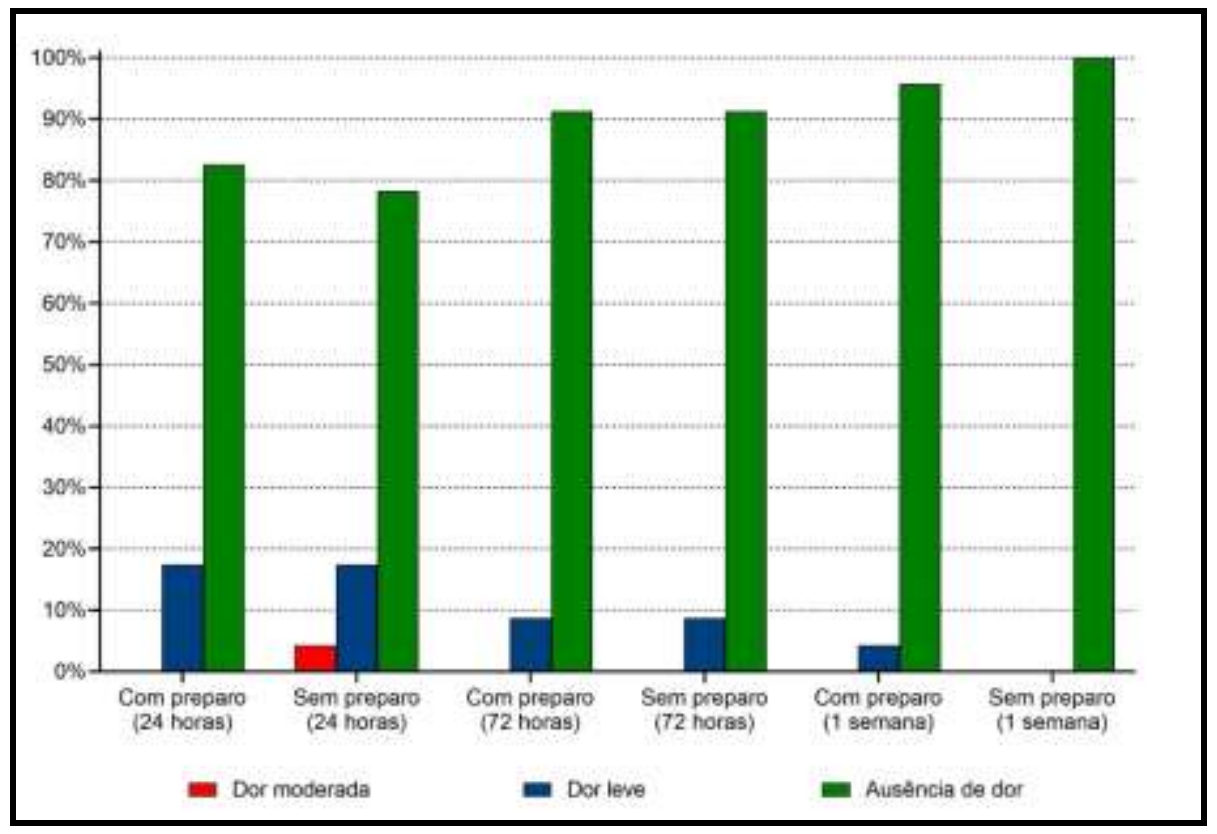

Fonte: Autores.

O teste de Kruskal-Wallis mostrou que nos pacientes onde foi realizado previamente o preparo dos terços cervical e médio não houve diferenças estatísticas entre o uso de analgésicos nos diferentes tempos experimentais $(\mathrm{p}=0.611)$. $\mathrm{O}$ mesmo teste aplicado ao grupo de pacientes onde não foi realizado o preparo prévio também não revelou diferenças significantes entre os resultados ( $\mathrm{p}=0.062)$ (Tabela 1$)$.

O teste de Mann-Whitney aplicado aos dados obtidos em ambos os grupos experimentais revelou que nos tempos de 24 horas ( $\mathrm{p}=0.916), 72$ horas $(\mathrm{p}=0.744)$ e 1 semana $(\mathrm{p}=1.00)$, não houve diferença entre os tipos de preparo realizados no que diz respeito ao uso de analgésicos (Tabela 2).

Tabela 1. Resultados relativos à necessidade do uso de analgésicos nos grupos estudados* .

\begin{tabular}{|c|c|c|c|c|}
\hline \multirow[t]{3}{*}{ Grupos } & \multicolumn{3}{|c|}{ Tempo } & Valor de P \\
\hline & & & & Teste \\
\hline & 24 horas & 72 horas & 1 semana & Kruskal-Wallis \\
\hline Com preparo cervical & $1(1-3)$ & $1(1-3)$ & $1(1-3)$ & 0.611 \\
\hline Sem preparo cervical & $1(1-3)$ & $1(1-2)$ & $1(1-1)$ & 0.062 \\
\hline Teste de Mann-Whitney & 0.916 & 0.744 & 1.000 & \\
\hline
\end{tabular}

*Não foram encontradas diferenças estatísticas para nível de significância $\alpha=5 \%$. Fonte: Autores.

\section{Discussão}

Os pacientes que iniciam o tratamento endodôntico sem sintomas podem sentir dor após a obturação do sistema de canais radiculares (Harisson el al., 1983). A etiologia da dor pós-operatória está relacionada à presença de microrganismos, vitalidade pulpar, presença de dor no pré-operatório e também se houve patência do forame ou não durante o procedimento (Arias et al., 2009). Contudo, o presente estudo teve o cuidado de descartar fatores pré-existentes que poderiam afetar o 
objetivo principal do trabalho: avaliar a sensibilidade dolorosa em dentes tratados endodonticamente em sessão única com preparo prévio e sem preparo prévio para realizar a patência foraminal. A não manutenção da patência foraminal durante o preparo do canal radicular pode influenciar no pós-operatório da terapia endodôntica. A patência com instrumentos mecanizados apresentam uma menor modificação da trajetória original do canal quando comparado com o uso de instrumentos manuais (Berutti et al., 2009). Quando se trata de dentes não vitais, há um grau significante de menor dor quando a patência apical é mantida (Arias et al., 2009).

Este estudo realizou tratamentos endodônticos apenas em dentes com necrose pulpar e que o paciente não estivesse usando medicação em período de 10 dias antes da sessão. Esse critério teve por objetivo não mascarar os resultados e ter melhor controle do pós-operatório quanto ao grau de dor e a necessidade de uso de analgésico após a terapia endodôntica. A Escala Visual Analógica (EVA) foi utilizada para avaliar a dor e este método se mostrou eficiente e confiável para esse fim (Kersten et al., 2012).

O instrumento utilizado para o preparo do canal radicular foi o WaveOne Gold (NiTi M-Wire). A vantagem do uso de instrumentos reciprocantes incluem o tempo reduzido de trabalho, menor custo, menor fadiga dos instrumentos e a eliminação de possíveis contaminações cruzadas (agentes infecciosos) associadas ao uso único de instrumentos endodônticos (Shen et al., 2013, Yared et al., 2008). Uma das possibilidades da dor pós-operatória é a extrusão de debris. Tanto os sistemas rotatórios, quanto os reciprocantes, produzem debris (Burkein \& Shafer, 2011; Robinson et al., 2013).

O sistema reciprocante produz menos debris em comparação com o rotatório (Koçak et al., 2013). Quando comparados um sistema rotatório (Protaper Next) e dois reciprocantes (Reciproc - VDW e WaveOne - Dentsply Maillefer, Ballaigues, Switzerland) in vivo em tratamentos endodônticos com polpa vital, não houve diferença entre os grupos estudados. Os autores afirmaram que a cinemática padronizada e controlada usada durante o estudo pode ter contribuído para minimizar restos de debris ou extrusão destes, reduzindo assim a possível contribuição deste fator para a ocorrência de dor pós-operatória (Kherlakian et al., 2016).

O preparo convencional da terapia endodôntica é coroa-ápice. Sendo que deve se realizar uma desinfecção progressiva do terço cervical e médio com o fim de se chegar ao forame com menor carga microbiana intracanal. Dessa maneira, diminuindo a possibilidade de extrusão de microrganismos para o periápice e consequentemente não causar dor pósoperatória. Este estudo teve por hipótese nula que não haveria diferença entre os grupos CPP e SPP e esta foi aceita pois os resultados foram equivalentes.

Os tratamentos endodônticos foram realizados em sessão única neste estudo. Poucos pacientes fizeram o uso de analgésicos após a terapia endodôntica. Outros estudos mostraram que não houve diferença estatística entre tratamentos em sessão única e múltiplas sessões. Pacientes submetidos a uma única sessão podem experimentar uma frequência ligeiramente maior de edema e referir significativamente mais uso de analgésicos (Ehsani et al., 2008). Tratamentos endodônticos realizados em sessão única, com ou sem terapia fotodinâmica, não mostrou nenhuma diferença nos sintomas de dor entre os grupos em 24 h, 72 h e 1 semana (Barciela et al., 2019)

Outros estudos apresentam resultados semelhantes onde os prepares foram realizados em $0.0 \mathrm{~mm}$ e $1.0 \mathrm{~mm}$ aquém do ápice com ou sem ampliação foraminal. Os resultados mostraram que o desconforto ocorreu de forma leve dentro das 24 horas, mas nenhuma diferença estatística foi observada nos períodos de 72 horas e 1 semana (Cruz Junior et al., 2016). Os sistemas reciprocantes e rotatórios mostraram-se equivalentes quanto à incidência de dor pós-operatória e ingestão de medicação analgésica (Kherlakian et al., 2016). Outro fator importante que deve ser avaliado, é a presença ou não de radiolucência apical e tratar em sessão única ou múltiplas. Em períodos avaliados em 24 horas, 72 horas e 1 semana não houve diferenças entre dentes unirradiculares e multirradiculares com o sem lesão apical em terapia de sessão única (Raju et al., 2014). 
O uso de medicação pré-operatória é também um protocolo preconizado por muitos endodontistas, com o objetivo de se obter maior conforto pós-operatório ou não ter dor. Observou-se que o uso profilático do ibuprofeno e do acetaminofeno em dentes com lesão apical promoveu uma redução na dor pós-tratamento entre 4 horas (ibuprofeno) e 8-12 horas (acetaminofeno) comparados com grupo placebo (Ehsani et al., 2012). Neste estudo, o analgésico de escolha foi o ibuprofeno $600 \mathrm{mg}$ prescrito de 8/8 horas se houvesse dor. No período de 24 horas no grupo CPP, 1 paciente utilizou analgésico no período de 24 horas e 1 semana. No grupo SPP, 1 paciente utilizou o analgésico com uso descontínuo nas primeiras 24 horas e 72 horas e 1 semana nenhum paciente fez uso de medicamento.

\section{Conclusão}

Pôde-se concluir que a sensibilidade dolorosa pós-tratamento endodôntico em dentes unirradiculares, sem vitalidade pulpar com ou sem preparo prévio do terço cervical e médio do canal, apresentou comportamento equivalente.

\section{Referências}

Arias, A., Azabal, M., Hidalgo, J. J., \& José, C. (2009). Relationship between postendodontic pain, tooth diagnostic factors, and apical patency. Journal of endodontics, 35(2), 189-192.

Arias, A., de la Macorra, J. C., Hidalgo, J. J., \& Azabal, M. (2013). Predictive models of pain following root canal treatment: a prospective clinical study. International endodontic journal, 46(8), 784-793.

Barciela, B., da Silva Limoeiro, A. G., Bueno, C. E., Fernandes, S. L., Mandarini, D. R., Boer, N. C., ... \& Rocha, D. G. (2019). In vivo evaluation of painful symptomatology after endodontic treatment with or without the use of photodynamic therapy. Journal of conservative dentistry: JCD, 22 (4), 332.

Berutti, E., Cantatore, G., Castellucci, A., Chiandussi, G., Pera, F., Migliaretti, G., \& Pasqualini, D. (2009). Use of nickel-titanium rotary PathFile to create the glide path: comparison with manual preflaring in simulated root canals. Journal of Endodontics, 35(3), 408-412.

Buchanan, L. S. (1989). Management of the curved root canal. J Calif Dent Assoc, 17, 18-25.

Burklein S, Shafer E. Apically Extruded Debris with Reciprocating Single-File and Full-sequence Rotary Instrumentation Systems. J End. 2012;38(6):850-2.

Figini, L., Lodi, G., Gorni, F., \& Gagliani, M. (2008). Single versus multiple visits for endodontic treatment of permanent teeth: a Cochrane systematic review. Journal of Endodontics, 34(9), 1041-1047.

Franco, V., Fabiani, C., Taschieri, S., Malentacca, A., Bortolin, M., \& Del Fabbro, M. (2011). Investigation on the shaping ability of nickel-titanium files when used with a reciprocating motion. Journal of endodontics, 37(10), 1398-1401.

Haapasalo, M., Shen, Y., Qian, W., \& Gao, Y. (2010). Irrigation in endodontics. Dental Clinics, 54(2), 291-312.

Harrison, J. W., Baumgartner, J. C., \& Svec, T. A. (1983). Incidence of pain associated with clinical factors during and after root canal therapy. Part 2. Postobturation pain. Journal of endodontics, 9(10), 434-438.

Junior, J. A. C., Coelho, M. S., Kato, A. S., Vivacqua-Gomes, N., Fontana, C. E., Rocha, D. G. P., \& da Silveira Bueno, C. E. (2016). The effect of foraminal enlargement of necrotic teeth with the reciproc system on postoperative pain: a prospective and randomized clinical trial. Journal of endodontics, $42(1)$, 8-11.

Kersten, P., Küçükdeveci, A. A., \& Tennant, A. (2012). The use of the Visual Analogue Scale (VAS) in rehabilitation outcomes. Journal of rehabilitation medicine, 44(7), 609-610.

Kherlakian, D., Cunha, R. S., Ehrhardt, I. C., Zuolo, M. L., Kishen, A., \& da Silveira Bueno, C. E. (2016). Comparison of the incidence of postoperative pain after using 2 reciprocating systems and a continuous rotary system: a prospective randomized clinical trial. Journal of Endodontics, $42(2)$, 171-176.

Koçak, S., Koçak, M. M., Sağlam, B. C., Türker, S. A., Sağsen, B., \& Er, Ö. (2013). Apical extrusion of debris using self-adjusting file, reciprocating singlefile, and 2 rotary instrumentation systems. Journal of endodontics, 39(10), 1278-1280.

Larsen, C. M., Watanabe, I., Glickman, G. N., \& He, J. (2009). Cyclic fatigue analysis of a new generation of nickel titanium rotary instruments. Journal of endodontics, 35(3), 401-403.

Marshall, F. J., \& Pappin J. (1980). A crown-down pressureless preparation root canal enlargement technique. Oregon Health University.

Myles, P. S., Troedel, S., Boquest, M., \& Reeves, M. (1999). The pain visual analog scale: is it linear or nonlinear?Anesthesia \& Analgesia, 87(6), 1517.

Pruett, J. P., Clement, D. J., \& Carnes Jr, D. L. (1997). Cyclic fatigue testing of nickel-titanium endodontic instruments. Journal of endodontics, $23(2)$, 77-85.

Raju, T. B. V. G., Seshadri, A., Vamsipavani, B., Abhilash, K., Subhash, A. V., \& Kumari, K. H. (2014). Evaluation of pain in single and multi rooted teeth treated in single visit endodontic therapy. Journal of international oral health: JIOH, 6(1), 27. 
Research, Society and Development, v. 10, n. 7, e41310716677, 2021 (CC BY 4.0) | ISSN 2525-3409 | DOI: http://dx.doi.org/10.33448/rsd-v10i7.16677

Roane, J. B., Sabala, C. L., \& Duncanson Jr, M. G. (1985). The "balanced force" concept for instrumentation of curved canals. Journal of endodontics, 11(5), 203-211.

Robinson, J. P., Lumley, P. J., Cooper, P. R., Grover, L. M., \& Walmsley, A. D. (2013). Reciprocating root canal technique induces greater debris accumulation than a continuous rotary technique as assessed by 3-dimensional micro-computed tomography. Journal of Endodontics, 39(8), 1067-1070.

Sathorn, C., Parashos, P., \& Messer, H. (2008). The prevalence of postoperative pain and flare-up in single-and multiple-visit endodontic treatment: a systematic review. International endodontic journal, 41(2), 91-99.

Schilder, H. (2006). Filling root canals in three dimensions. Journal of endodontics, 32(4), 281-290.

Shen, Y., Zhou, H. M., Zheng, Y. F., Peng, B., \& Haapasalo, M. (2013). Current challenges and concepts of the thermomechanical treatment of nickeltitanium instruments. Journal of endodontics, 39(2), 163-172.

Silva, E. J. N. L., Menaged, K., Ajuz, N., Monteiro, M. R. F. P., \& de Souza Coutinho-Filho, T. (2013). Postoperative pain after foraminal enlargement in anterior teeth with necrosis and apical periodontitis: a prospective and randomized clinical trial. Journal of endodontics, 39(2), $173-176$.

Souza, R. A. (2000). Limpeza de forame e sua relação com a dor pós-operatória. Jornal Brasileiro de Endo/Perio, 1(3), 45-8.

Walia, H., Brantley, W. A., \& Gerstein, H. (1988). An initial investigation of the bending and torsional properties of Nitinol root canal files. Journal of endodontics, 14(7), 346-351.

Yared G. A 3-year Outcome of Endododontic Treatment Done with the RECIPROC® Single File Canal Preparation System. Munich, VDW; 2012.

You, S. Y., Kim, H. C., Bae, K. S., Baek, S. H., Kum, K. Y., \& Lee, W. (2011). Shaping ability of reciprocating motion in curved root canals: a comparative study with micro-computed tomography. Journal of endodontics, 37(9), 1296-1300. 